\title{
Stochastic Differential Equation on Extinction Probability
}

\author{
Agba, M. $\mathbf{E}^{1}$, Ugbebor, O. $\mathbf{O}^{2}$ \\ Department of Mathematics, F.C.E, Obudu, Nigeria \\ Department of Mathematics, University of Ibadan, Ibadan, Nigeria
}

\begin{abstract}
This study was designed to develop and apply a stochastic differential equation that proves and computes the probability of extinction of a giving family using factorization method. This method was used on the stochastic birth-death process equation to determine the probability generating function. The limiting values of the generating function $G(t, z)$ as $t \rightarrow \infty, z=0$ were taken to determine the extinction probability. Forty (40) families were studied and purposefully administered (by one-on-one interaction) to heads of families using the ten (10) wards in Bekwarra Local Government Area, Cross River State to ascertain the birth rate, date rate and extinction probability.
\end{abstract}

Keywords: Extinction probability, Stochastic differential equation, Birth-death process

\section{Introduction}

The probability of extinction is an important phenomenon in population dynamics and the study of evolution. The major factors affecting extinction are the birth and death respectively (Dzaan, Onah, and Kimbir, 2009; Scott, 2006; and Omotosho 2014).

Researchers have studied birth-death processes using certain mathematical models without employing the factorization method which is tractable in analysis the stochastic process in extinction probability.

\section{Methodology of stochastic differential equation for birth-death process}

The factorization method is used on the stochastic birthdeath process equation to determine the probability generating function.

Consider the stochastic birth-death process equation given as;

$\frac{\partial}{\partial t} \mathrm{p}_{\mathrm{n}}(\mathrm{t})=\lambda(\mathrm{n}-1) \mathrm{p}_{\mathrm{n}-1}(\mathrm{t})+\mu(\mathrm{n}+1) \mathrm{p}_{\mathrm{n}+1}(\mathrm{t})-(\lambda+\mu) n \mathrm{p}_{\mathrm{n}}(\mathrm{t})$

[Where, $\mathrm{n}$ is an integer and the probability for negative $\mathrm{n}$ is assumed to be zero, $\left(\mathrm{p}_{\mathrm{n}}(\mathrm{t})=0\right.$ for $\mathrm{n}<0$ for non-negative initial population size $n(0)>0)$, (Fugo, 2009).

The probability generating function for a probability distribution $p_{n}(\mathrm{t})$ is defined:

$\mathrm{G}(\mathrm{t}, \mathrm{z})=\sum_{n=o}^{\infty} p_{n}(\mathrm{t}) \mathrm{z}^{\mathrm{n}}$

We have (2) as; $\frac{\partial}{\partial t} \mathrm{G}(\mathrm{t}, \mathrm{z})=\sum_{n=0}^{\infty} z^{n} \frac{\partial}{\partial t} \mathrm{p}_{\mathrm{n}}(\mathrm{t})$

But,

$\mathrm{p}_{\mathrm{n}}^{\prime}(\mathrm{t})=\lambda(\mathrm{n}-1) \mathrm{p}_{\mathrm{n}-1}(\mathrm{t})+\mu(\mathrm{n}+1) \mathrm{p}_{\mathrm{n}+1}(\mathrm{t})-(\lambda+\mu) n \mathrm{p}_{\mathrm{n}}(\mathrm{t})$

Then,

$\frac{\partial G}{\partial t}(\mathrm{t} ; \mathrm{z})=\sum_{n=0}^{\infty} \mathrm{z}^{\mathrm{n}} \mathrm{p}_{\mathrm{n}}^{\prime}(\mathrm{t})=\lambda \sum_{n=0}^{\infty}(n-1) \mathrm{p}_{\mathrm{n}}-1(\mathrm{t}) \mathrm{z}^{\mathrm{n}}+$

$\mu \sum(n+1) \mathrm{p}_{\mathrm{n}+1}(\mathrm{t}) \mathrm{z}^{n}-(\lambda+\mu) \sum n \mathrm{p}_{\mathrm{n}}(\mathrm{t}) \mathrm{z}^{\mathrm{n}}$

$=\left[\left(\mathrm{p}_{1}(\mathrm{t}) \mathrm{z}^{2}+2 \mathrm{p}_{2}(\mathrm{t}) \mathrm{z}^{3}+3 \mathrm{p}_{3}(\mathrm{t}) \mathrm{z}^{4}\right)+\ldots+\right]$

$+\mu\left[\mathrm{p}_{1}(\mathrm{t}) \mathrm{z}^{0}+2 p_{2}(\mathrm{t}) \mathrm{z}+3 \mathrm{p}_{3}(\mathrm{t}) \mathrm{z}^{2}+4 p_{4}(\mathrm{t}) \mathrm{z}^{3}\right.$

$+\ldots+]$

$+(\lambda+\mu)\left[-\mathrm{p}_{1}(\mathrm{t}) \mathrm{z}-2 \mathrm{p}_{2}(\mathrm{t}) \mathrm{z}^{2}-3 \mathrm{p}_{3}(\mathrm{t}) \mathrm{z}^{3}-\ldots\right]+$ $=\left[\lambda \mathrm{p}_{1}(\mathrm{t}) \mathrm{z}^{2}+\mu \mathrm{p}_{1}(\mathrm{t}) \mathrm{z}^{0}-(\lambda+\mu) \mathrm{p}_{1}(\mathrm{t}) \mathrm{z}\right]$

$+\left[2 \lambda \mathrm{p}_{2}(\mathrm{t}) \mathrm{z}^{3}+2 \mu \mathrm{p} 2(\mathrm{t}) \mathrm{z}-(\lambda+\mu) 2 \mathrm{p}_{2}(\mathrm{t}) \mathrm{z}^{2}\right]$

$+\left[3 \lambda \mathrm{p}_{3}(\mathrm{t}) \mathrm{z}^{4}+3 \mu \mathrm{p}_{3}(\mathrm{t}) \mathrm{z}^{2}-(\lambda+\mu) 3 \mathrm{p}_{3}(\mathrm{t}) \mathrm{z}^{3}\right]$

$=\left[\lambda \mathrm{p}_{1}(\mathrm{t}) \mathrm{z}^{2}+\mu \mathrm{p}_{1}(\mathrm{t}) \mathrm{z}^{0}-\lambda \mathrm{p}_{1}(\mathrm{t}) \mathrm{z}-\mu \mathrm{p}_{1}(\mathrm{t}) \mathrm{z}\right]$

$+\left[2 \lambda \mathrm{p}_{2}(\mathrm{t}) \mathrm{z}^{3}+2 \mu \mathrm{p}_{2}(\mathrm{t}) \mathrm{z}-2 \lambda \mathrm{p}_{2}(\mathrm{t}) \mathrm{z}^{2}-2 \mu \mathrm{p}_{2}(\mathrm{t}) \mathrm{z}^{2}\right]$

$+\left[3 \lambda \mathrm{p}_{3}(\mathrm{t}) \mathrm{z}^{4}+3 \mu \mathrm{p}_{3}(\mathrm{t}) \mathrm{z}^{2}-3 \lambda \mathrm{p}_{3}(\mathrm{t}) \mathrm{z}^{3}-3 \mu \mathrm{p}_{3}(\mathrm{t}) \mathrm{z}^{3}\right]+\ldots$

Rearrange the summation and factorise we now have:

$\lambda_{1} \mathrm{p}_{1}(\mathrm{t}) \mathrm{z}^{2}+\mu \mathrm{p}_{1}(\mathrm{t}) \mathrm{z}^{0}-\lambda \mathrm{p}_{1}(\mathrm{t}) \mathrm{z}-\mu \mathrm{p}_{1}(\mathrm{t}) \mathrm{z}=\lambda_{1} p_{1}(t) \mathrm{z}^{2}+$ $\mu \mathrm{p}_{1}(\mathrm{t}) \mathrm{z}^{0}-\lambda \mathrm{p}_{1}(\mathrm{t}) \mathrm{z}-\mu \mathrm{p}_{1}(\mathrm{t}) \mathrm{z}$

$=\lambda \mathrm{p}_{1}(\mathrm{t}) \mathrm{z}^{2}-\lambda \mathrm{p}_{1}(\mathrm{t}) \mathrm{z}+\mu \mathrm{p}_{1}(\mathrm{t}) \mathrm{z}^{0}-\mu \mathrm{p}_{1}(\mathrm{t}) \mathrm{z}$

$$
=\lambda \mathrm{p}_{1}(\mathrm{t}) \mathrm{z}(\mathrm{z}-1)+\mu \mathrm{p}_{1}(\mathrm{t})(1-\mathrm{z})
$$

Also,

$$
\begin{aligned}
& 2 \lambda \mathrm{p}_{2}(\mathrm{t}) \mathrm{z}^{3}+2 \mu \mathrm{p}_{2}(\mathrm{t}) \mathrm{z}-2 \lambda \mathrm{p}_{2}(\mathrm{t}) \mathrm{z}^{2}-2 \mu \mathrm{p}_{2}(\mathrm{t}) \mathrm{z}^{2} \\
& =2 \lambda \mathrm{p}_{2}(\mathrm{t}) \mathrm{z}^{3}-2 \lambda \mathrm{p}_{2}(\mathrm{t}) \mathrm{z}^{2}+2 \mu \mathrm{p}_{2}(\mathrm{t}) \mathrm{z}-2 \mu \mathrm{p}_{2}(\mathrm{t}) \mathrm{z}^{2} \\
& \quad=2 \lambda \mathrm{p}_{2}(\mathrm{t}) \mathrm{z}^{2}[\mathrm{z}-1]+2 \mu \mathrm{p}_{2}(\mathrm{t}) \mathrm{z}[1-\mathrm{z}] \\
& \begin{array}{r}
\text { similarly, } \\
3 \lambda \mathrm{p}_{3} \mathrm{z}^{4}+3 \mu \mathrm{p}_{3}(\mathrm{t}) \mathrm{z}^{2}-3 \lambda \mathrm{p}_{3}(\mathrm{t}) \mathrm{z}^{3}-3 \mu \mathrm{p}_{3}(\mathrm{t}) \mathrm{z}^{3} \\
=3 \lambda \mathrm{p}_{3}(\mathrm{t}) \mathrm{z}^{3}[\mathrm{z}-1]+3 \mu \mathrm{p}_{3}(\mathrm{t}) \mathrm{z}^{2}[1-\mathrm{z}]
\end{array}
\end{aligned}
$$

Sum equations (5), (6) and (7), generated from equation (4)

$$
\left[\lambda \mathrm{p}_{1}(\mathrm{t}) \mathrm{z}(\mathrm{z}-1)+\mu \mathrm{p}_{1}(\mathrm{t})(1-\mathrm{z})\right]
$$

$+\left[2 \lambda \mathrm{p}_{2}(\mathrm{t}) \mathrm{z}^{2}(\mathrm{z}-1)+2 \mu \mathrm{p}_{2}(\mathrm{t}) \mathrm{z}(1-\mathrm{z})\right]$

$+\left[3 \lambda \mathrm{p}_{3}(\mathrm{t}) \mathrm{z}^{3}(\mathrm{z}-1)+3 \mu \mathrm{p}_{3}(\mathrm{t}) \mathrm{z}^{2}(1-\mathrm{z})\right]$

$=\lambda \mathrm{z}(\mathrm{z}-1)\left[\mathrm{p}_{1}(\mathrm{t})+2 \mathrm{p}_{2}(\mathrm{t}) \mathrm{z}+3 \mathrm{p}_{3}(\mathrm{t}) \mathrm{z}^{2}\right]+$

$\mu(1-\mathrm{z})\left[\mathrm{p}_{1}(\mathrm{t})+2 \mathrm{p}_{2}(\mathrm{t}) \mathrm{z}+3 \mathrm{p}_{3}(\mathrm{t}) \mathrm{z}^{2}\right]+\ldots$

$=[\lambda \mathrm{z}(\mathrm{z}-1)+\mu(1-\mathrm{z})]\left[p_{1}(\mathrm{t})+2 p_{2}(\mathrm{t}) \mathrm{z}+3 p_{3}(\mathrm{t}) z^{2}+\ldots+\right]$

$=[\lambda \mathrm{z}(\mathrm{z}-1)+\mu(1-\mathrm{z})] \sum_{n=0}^{\infty} n \mathrm{p}_{\mathrm{n}}(\mathrm{t}) \mathrm{z}^{\mathrm{n}-1}$

$=\left(\lambda \mathrm{z}^{2}-\lambda \mathrm{z}-\mu \mathrm{z}+\mu\right) \sum_{n=0}^{\infty} n \mathrm{p}_{\mathrm{n}}(\mathrm{t}) \mathrm{z}^{\mathrm{n}-1}$

$\frac{\partial G}{\partial t}(\mathrm{t}, \quad \mathrm{z})=\left[\lambda \mathrm{z}^{2}-\left(\lambda \mathrm{z}^{2}+\mu\right) \mathrm{z}+\mu\right] \sum_{n=0}^{\infty} n \mathrm{p}_{\mathrm{n}}(\mathrm{t}) \mathrm{z}^{\mathrm{n}}-1=$ $\sum_{n=0}^{\infty} z_{\mathrm{n}} \frac{\partial}{\partial t} p_{\mathrm{n}}(\mathrm{t})$

Therefore,

$\sum_{n=0}^{\infty} z^{n} \mathrm{p}_{\mathrm{n}}^{\prime}(\mathrm{t})=\left\{\lambda \mathrm{z}^{2}-(\lambda+\mu) \mathrm{z}+\mu\right\} \sum_{n=0}^{\infty} n \mathrm{p}_{\mathrm{n}}(\mathrm{t}) \mathrm{z}^{\mathrm{n}-1}$

We write,

$\left[\lambda \mathrm{z}^{2}-(\lambda+\mu) \mathrm{z}+\mu\right] \sum_{n=0}^{\infty} n \mathrm{p}_{\mathrm{n}}(\mathrm{t}) \mathrm{z}^{\mathrm{n}-1}=\frac{\partial G}{\partial t}(\mathrm{t}, \mathrm{z})$

Or

$$
\frac{\partial G}{\partial t}(\mathrm{t}, \mathrm{z})=\left[\lambda \mathrm{z}^{2}-(\lambda+\mu) \mathrm{z}+\mu\right] \sum_{n=0}^{\infty} n \mathrm{p}_{\mathrm{n}}(\mathrm{t}) \mathrm{z}^{\mathrm{n}-1}
$$


Recall that,

$\frac{\partial G}{\partial z}(\mathrm{t}, \mathrm{z})=\sum_{n=0}^{\infty} n \mathrm{p}_{\mathrm{n}}(\mathrm{t}) \mathrm{z}^{\mathrm{n}-1}$, for $\mathrm{G}(\mathrm{t}, \mathrm{z})=\sum_{n=0}^{\infty} n \mathrm{p}_{\mathrm{n}}(\mathrm{t}) \mathrm{z}^{\mathrm{n}}$

Then, for the linear birth and death process in equation (8) we have;

$$
\frac{\partial G}{\partial t}(\mathrm{t}, \mathrm{z})=\left[\lambda \mathrm{z}^{2}-(\mu+\lambda) \mathrm{z}+\mu\right] \frac{\partial G}{\partial z}(\mathrm{t}, \mathrm{z})
$$

Equating to zero :

Let

$$
\frac{\partial G}{\partial t}(\mathrm{t}, \mathrm{z})-\left[\lambda \mathrm{z}^{2}-(\mu+\lambda) \mathrm{z}+\mu\right] \frac{\partial G}{\partial z}(\mathrm{t}, \mathrm{z})=0
$$

$$
\begin{aligned}
& a(z, t, G)=-\left[\lambda z^{2}-(\mu+\lambda) \mathrm{z}+\mu\right] \frac{\partial G}{\partial z}(\mathrm{t}, \mathrm{z}) \\
& b(z, t, G)=1 \\
& c(z, t, G)=0
\end{aligned}
$$

The partial differential equation is of the form;

Written as,

$$
b \frac{\partial G}{\partial t}+a \frac{\partial G}{\partial z}=\mathrm{c}
$$

$$
b \frac{\partial G}{\partial t}+a \frac{\partial G}{\partial z}=0
$$

With the initial and boundary conditions for $\mathrm{N}(0)$ at time $\mathrm{t}=$ 0 , the initial condition is $P_{\mathrm{n}}(0)$ for $\mathrm{n} \neq N(0)$ and $\mathrm{P}_{\mathrm{N}(0)}=1$, that is

$$
\mathrm{G}(\mathrm{t}, \mathrm{z})=\sum_{n} P_{\mathrm{n}}(\mathrm{t}) \mathrm{z}^{\mathrm{n}}=\mathrm{z}^{\mathrm{n}(0)},
$$

The boundary condition determines $\mathrm{G}(\mathrm{t}, 0)$ and $\mathrm{G}(\mathrm{t}, 1)$ is expressed as;

$$
\begin{aligned}
\mathrm{G}(\mathrm{t}, \mathrm{z}) & =P_{0} \\
\mathrm{G}(\mathrm{t}, 1) & =\sum_{n} P_{\mathrm{n}}(\mathrm{t}) \mathrm{z}=1
\end{aligned}
$$

$p_{o}$ is undermined at this point. Solving $p_{n}(t)$ (t) is now reduced to solving the partial differential equation of $\mathrm{G}(\mathrm{t}, \mathrm{z})$ in (9), with condition (10), (11) and (12).

Consider the partial differential equation in $\mathrm{G}(\mathrm{t}, \mathrm{z})$ involving $\frac{\partial G}{\partial t}(\mathrm{t}, \mathrm{z})$ and $\frac{\partial G}{\partial t}(t, z),($ Fugo, 2009):

The auxiliary equation is given as;

$$
\frac{\partial x}{1}=\frac{\partial y}{1}=\frac{\partial i}{1}
$$

Adopting similar pattern, we have the auxiliary equation as;

$$
\begin{gathered}
\frac{-\partial z}{\lambda z^{2+\mu-(\lambda+\mu) z}}=\frac{\partial t}{1}=\frac{\partial G}{0} \\
\frac{-\partial z}{(1-z)(\mu-\lambda z)}=\frac{\partial t}{1}=\frac{\partial G}{0}
\end{gathered}
$$

Integrate the equation

$$
\int \frac{1}{(1-z)(\mu-\lambda z)} \partial z=-\int 1 \partial t
$$

First, resolve LHS of (13)

$$
\frac{1}{(1-z)(\mu-\lambda z)} \quad=\frac{A}{(1-Z)}+\frac{B}{(\mu-\lambda)}
$$

Multiply all through by $(1-\mathrm{z})(\mu-\lambda \mathrm{z})$

$$
1=\mathrm{A}(\mu-\lambda \mathrm{z})+\mathrm{B}(1-\mathrm{z})
$$

putting $\mathrm{z}=1$ in (14), we have,

Putting $\mathrm{z}=0$,

$$
\begin{aligned}
& 1=\mathrm{A}(\mu-\lambda) \\
& \mathrm{A}=\frac{1}{\mu-\lambda}
\end{aligned}
$$

$$
\begin{aligned}
& 1=\mathrm{A} \mu+B \\
& \mathrm{~B}=1-A \mu
\end{aligned}
$$

$$
\begin{gathered}
=1-\frac{\mu}{\mu-\lambda} \\
=\frac{-\lambda}{\mu-\lambda}
\end{gathered}
$$

Therefore,

$$
\frac{1}{(1-z)(\mu-\lambda z)} \equiv \frac{1}{\mu-\lambda}\left[\frac{1}{1-z}-\frac{\lambda}{\mu-\lambda z}\right]
$$

we have (13) as,

$$
\begin{gathered}
\int \frac{1}{\mu-\lambda}\left(\frac{1}{1-\mathrm{z}}-\frac{\lambda}{\mu-\lambda \mathrm{z}}\right) \partial z=-\int \partial t \\
\frac{1}{\mu-\lambda}[-\operatorname{In}(1-z)+\operatorname{In}(\mu-\lambda \mathrm{z}]=-t \\
\operatorname{In}\left(\frac{\mu-\lambda \mathrm{z}}{1-\mathrm{z}}\right)=e^{-(\mu-\lambda) t} \\
\frac{\mu-\lambda \mathrm{z}}{(1-\mathrm{z}) e^{(\lambda-\mu) t}}=\mathrm{K}_{1} \\
\mathrm{~K}_{1}=\frac{(\mu-\lambda \mathrm{z}) \mathrm{e}^{-(\lambda-\mu) \mathrm{t}}}{(1-z)} \\
\frac{\partial t}{1}=\frac{\partial G}{0} \\
0 \partial t=\int_{\mathrm{X}} \partial G \\
\mathrm{~K}_{2}=\mathrm{X}
\end{gathered}
$$

The general solution is,

$$
\mathrm{G}(\mathrm{t}, \mathrm{z})=\mathrm{X}(\mathrm{V})
$$

$\mathrm{X}$ can be any function of,

$$
\mathrm{V}=e^{-(\lambda-\mu) t}\left(\frac{\mu-\lambda \mathrm{z}}{1-\mathrm{z}}\right),
$$

for time $(\mathrm{t})=0$ and $\lambda \neq \mu, \sum_{n=1}^{\infty} p_{n}(0)$

we solve $G(t, z)$ in the general solution

$$
\begin{gathered}
G(t, z)=e^{-(\lambda-\mu) t}\left(\frac{\mu-\lambda z}{1-z}\right), \\
G(0, z)=x\left(\frac{\mu-\lambda z}{1-z}\right) \\
G(0, z)=\sum_{n=0}^{\infty} p_{n}(o) z^{n}=z^{n}
\end{gathered}
$$

for $\mathrm{t}=0$ (15) becomes,

make $\mathrm{z}$ the subject in (16)

$$
\begin{gathered}
\mathrm{V}(1-z)=\mu-\lambda \mathrm{z} \\
\mathrm{V}-\mathrm{Vz}=\mu-\lambda \mathrm{z} \\
\lambda \mathrm{z}-\mathrm{Vz}=\mu-V \\
z(\lambda-\mathrm{V})=\mu-V \\
\mathrm{z}=\frac{\mu-V}{\lambda-\mathrm{V}},
\end{gathered}
$$

substitute (16) into (17) for,

Yields

$$
\mathrm{G}(0, \mathrm{z})=\mathrm{X}\left(\left.\mathrm{V}\right|_{\mathrm{t}=0}\right)=\mathrm{z}^{\mathrm{N}(0)}
$$

$$
\mathrm{G}(0, \mathrm{z})=\mathrm{X}\left(\left.\mathrm{V}\right|_{\mathrm{t}=0}\right)=\left(\frac{\mu-\left.V\right|_{t=O}}{\lambda-\left.V\right|_{t=o}}\right)^{N(o)}
$$

$\mathrm{X}$ is determined in it functional form by, $\mathrm{G}(\mathrm{t}, \mathrm{z})=\mathrm{X}(\mathrm{V})$ for $\mathrm{G}(\mathrm{t}, 1)=\mathrm{X}(0), \mathrm{z}=1$ and $\mathrm{t} \geq 0$.

Applying the uniqueness of solution of partial differential equation, $G(t, z)$ is evaluated, hence

$$
\begin{array}{r}
\mathrm{G}(\mathrm{t}, \mathrm{z})=\mathrm{z}^{\mathrm{N}(0)} \\
=\left(\frac{\mu-V}{\lambda-V}\right)^{N(o)}
\end{array}
$$

Substitute (16) in (19), 
International Journal of Science and Research (IJSR)

ISSN (Online): 2319-7064

Index Copernicus Value (2016): 79.57 | Impact Factor (2015): 6.391

$$
\begin{array}{r}
=\frac{\mu-\frac{\mu-\lambda \mathrm{z}}{1-\mathrm{z}} e^{-(\lambda-\mu) t}}{\lambda-\frac{\mu-\lambda \mathrm{z}}{1-\mathrm{z}} e^{-(\lambda-\mu) t}} \\
\therefore \mathrm{G}(\mathrm{t}, \mathrm{z})=\left[\frac{\mu-(1-z)-(\mu-\lambda z) e^{-(\lambda-\mu) t}}{\lambda(1-z)-(\mu-\lambda z) e^{-(\lambda-\mu) t}}\right]^{N(o)}
\end{array}
$$

Equation (20) is the probability generating function, Van Doorn (2004).

The extinction probability is derived from (20) by letting population size $n=0$ at time $p_{0}(t)$ and is given by $G(t, 0)$.

In the Birth - Death process, equation (20) yields:

$$
\begin{gathered}
\mathrm{p}_{0}(\mathrm{t})=\mathrm{G}(\mathrm{t}, 0)=\left[\frac{\mu-\mu e^{-(\lambda-\mu) t}}{\lambda-\mu e^{-(\lambda-\mu) t}}\right]^{N(o)} \\
\text { for, } \lambda>\mu \\
\mathrm{p}_{0}(\mathrm{t} \rightarrow \infty)=\lim _{t=\infty} G(0, t)=\left(\frac{\mu}{\lambda}\right)^{N(0)}<1, \\
\quad \lambda<\mu \\
\mathrm{p}_{0}(\mathrm{t} \rightarrow \infty)=\lim _{t=\infty} G(0, t)=1,
\end{gathered}
$$

If the birth rate is less than the death rate i.e, $\lambda<\mu$ the populations go extinct and if the birth rate is larger than the

\begin{tabular}{|c|c|c|c|c|c|c|c|c|c|c|c|c|}
\hline $\mathrm{S} / \mathrm{N}$ & WARD & WIFE & $\mathrm{N}_{\mathrm{D}}$ & $\mathrm{N}_{\mathrm{A}}$ & $\mathrm{T}_{\mathrm{B}}$ & $T_{D}$ & $\mathrm{~T}_{\mathrm{A}}$ & $T_{E}$ & $\bar{X}$ & $\lambda$ & $\underline{\mu}$ & $P_{E}$ \\
\hline 1 & Otukpuru & 1 & 1 & 2 & 5 & 1 & 6 & 4 & 2.500 & 833 & 167 & 0.200 \\
\hline 2 & Ibiaragidi & 3 & 0 & 4 & 16 & 2 & 18 & 14 & 1.333 & 889 & 111 & 0.125 \\
\hline 3 & Ugboro & 2 & 1 & 2 & 10 & 1 & 11 & 9 & 1.430 & 909 & 91 & 0.100 \\
\hline 4 & Ibiaragidi & 2 & 1 & 3 & 11 & 2 & 13 & 9 & 1.570 & 846 & 154 & 0.182 \\
\hline 5 & Ukpah & 1 & 0 & 5 & 9 & 3 & 12 & 6 & 2.250 & 750 & 250 & 0.333 \\
\hline 6 & Nyanya & 2 & 0 & 1 & 3 & 0 & 3 & 3 & 1.500 & 1000 & 0 & 0.000 \\
\hline 7 & Ukpah & 3 & 1 & 3 & 18 & 2 & 20 & 16 & 1.286 & 900 & 100 & 0.111 \\
\hline 8 & Gakem & 1 & 0 & 3 & 6 & 2 & 8 & 4 & 2.000 & 750 & 250 & 0.333 \\
\hline 9 & Ibiaragidi & 4 & 3 & 8 & 17 & 7 & 24 & 10 & 2.800 & 708 & 292 & 0.412 \\
\hline 10 & Ukpah & 3 & 1 & 3 & 6 & 1 & 7 & 5 & 3.00 & 857 & 143 & 0.170 \\
\hline 11 & Gakem & 3 & 1 & 4 & 8 & 3 & 11 & 5 & 2.670 & 727 & 273 & 0.376 \\
\hline 12 & Abouchiche & 4 & 3 & 8 & 17 & 7 & 24 & 10 & 2.800 & 708 & 292 & 0.412 \\
\hline 13 & Ibiaragidi & 2 & 0 & 7 & 13 & 3 & 16 & 10 & 2.170 & 813 & 188 & 0.231 \\
\hline 14 & Abuochiche & 3 & 1 & 11 & 21 & 3 & 24 & 18 & 2.100 & 875 & 125 & 0.143 \\
\hline 15 & Afrike 1 & 1 & 0 & 4 & 15 & 2 & 17 & 13 & 1.364 & 882 & 118 & 0.134 \\
\hline 16 & Nyanya & 2 & 1 & 7 & 13 & 2 & 15 & 11 & 2.600 & 867 & 133 & 0.153 \\
\hline 17 & Ugboro & 3 & 3 & 7 & 38 & 11 & 42 & 27 & 1.36 & 776 & 224 & 0.031 \\
\hline 18 & Abuochiche & 4 & 0 & 3 & 12 & 1 & 13 & 11 & 1.330 & 923 & 77 & 0.083 \\
\hline 19 & Otukpuru & 4 & 2 & 5 & 12 & 2 & 14 & 10 & 2.400 & 857 & 143 & 0.167 \\
\hline 20 & Beten & 7 & 2 & 10 & 62 & 3 & 65 & 59 & 1.240 & 954 & 46 & 0.048 \\
\hline 21 & Ukpah & 3 & 2 & 4 & 10 & 3 & 13 & 7 & 2.500 & 769 & 231 & 0.300 \\
\hline 22 & Nyanya & 4 & 0 & 3 & 13 & 1 & 14 & 12 & 1.300 & 929 & 71 & 0.076 \\
\hline 23 & Otukpuru & 3 & 2 & 4 & 12 & 5 & 17 & 7 & 2.400 & 706 & 294 & 0.416 \\
\hline 24 & Beten & 2 & 0 & 0 & 3 & 0 & 3 & 3 & 1.000 & 1000 & 0 & 0.000 \\
\hline 25 & Ibiaragidi & 8 & 0 & 4 & 12 & 3 & 15 & 9 & 1.50 & 800 & 200 & 0.250 \\
\hline 26 & Afrike II & 3 & 1 & 3 & 7 & 2 & 9 & 5 & 2.330 & 778 & 222 & 0.285 \\
\hline 27 & Otukpuru & 4 & 4 & 8 & 20 & 7 & 27 & 13 & 2.500 & 741 & 259 & 0.350 \\
\hline 28 & Beten & 5 & 2 & 12 & 25 & 6 & 31 & 19 & 2.27 & 806 & 194 & 0.241 \\
\hline 29 & Afrike II & 4 & 4 & 12 & 30 & 8 & 38 & 22 & 2.140 & 789 & 211 & 0.267 \\
\hline 30 & Ukpah & 5 & 5 & 20 & 36 & 8 & 44 & 26 & 4.000 & 818 & 182 & 0.222 \\
\hline 31 & Afrike I & 3 & 1 & 5 & 11 & 4 & 15 & 7 & 2.200 & 733 & 267 & 0.364 \\
\hline 32 & Afrike II & 3 & 1 & 10 & 22 & 9 & 31 & 13 & 2.000 & 710 & 290 & 0.408 \\
\hline 33 & Nyanya & 7 & 4 & 18 & 40 & 14 & 54 & 36 & 2.220 & 741 & 259 & 0.350 \\
\hline 34 & Abouchiche & 4 & 1 & 7 & 14 & 4 & 18 & 10 & 2.300 & 778 & 222 & 0.129 \\
\hline 35 & Ugboro & 8 & 3 & 54 & 70 & 8 & 78 & 62 & 5.380 & 897 & 103 & 0.145 \\
\hline 36 & Beten & 41 & 1 & 4 & 10 & 2 & 12 & 8 & 2.000 & 833 & 167 & 0.200 \\
\hline 37 & Gakem & 9 & 6 & 25 & 65 & 9 & 85 & 45 & 1.910 & 765 & 235 & 0.307 \\
\hline 38 & Afrike I & 7 & 4 & 15 & 31 & 11 & 42 & 20 & 2.580 & 738 & 262 & 0.355 \\
\hline 39 & Afrike II & 8 & 3 & 28 & 44 & 4 & 48 & 40 & 3.40 & 917 & 83 & 0.091 \\
\hline 40 & Beten & 6 & 3 & 14 & 30 & 12 & 42 & 18 & 2.310 & 909 & 286 & 0.315 \\
\hline
\end{tabular}
death rate $(\lambda>\mu)$ the populations can go extinct with nonzero probability $\left(\frac{\mu}{\lambda}\right)^{N(0)}$

For stochastic process, the probability of extinction is always positive and population is never free from extinction in the stochastic world

Table 1: Invaluable information arising from Locality

Where,

$N_{D}=$ number of individuals who died without offspring, $N_{A}$ $=$ number of individuals who are alive without offspring, $T_{B}=$ total number of births, $T_{D}=$ total number of death, $T_{E}=$ total number of events, $T_{A}=$ total number of individual who are alive, $\bar{x}=$ mean number of individuals, $\lambda=$ birth rate per thousands, $\mu=$ death rate per thousands and $p_{k}=$ extinction probability.

Evaluating extinction probability in the first family with number of birth 5 and death 1 .

Let

Volume 6 Issue 12, December 2017 www.ijsr.net

Licensed Under Creative Commons Attribution CC BY 


\section{International Journal of Science and Research (IJSR)}

ISSN (Online): 2319-7064

Index Copernicus Value (2016): 79.57 | Impact Factor (2015): 6.391

$$
\mu=\frac{d}{b+d} \text {. (1000) }
$$

Where. $\lambda=$ birth rate, $\mu=$ death rate, $\mathrm{b}=$ number of births in the family,

$\mathrm{d}=$ number of death in the family.

\section{Problem 1}

Let $b=5$ and $d=1$ in the first family, then;

$$
\begin{gathered}
\mu=\frac{d}{b+d} \cdot(1000) \\
=\frac{5}{5+1} \cdot(1000) \\
=\frac{5}{6} \cdot(1000) \\
=\frac{5000}{6} \\
=833 \\
\mu=\frac{d}{b+d} \cdot(1000) \\
=\frac{1}{5+1} \cdot(1000)
\end{gathered}
$$

$=\frac{1000}{6}=167$

The probability of extinction (3.3.2) for the family is given by:

$$
\begin{gathered}
P_{E}=\left(\frac{\mu}{\lambda}\right)^{n_{o}} \\
=\left(\frac{107}{833}\right)^{1} \\
=0.200
\end{gathered}
$$

$\bar{X}=$ The mean number of offspring $=\frac{\text { Total number of birt hs }}{\text { Number of fertile parents }}$

\section{Problem 2}

$$
\begin{aligned}
& \quad=\frac{T_{B}}{T_{B}-\left(N_{D}+N_{A}\right)} \\
& =\frac{5}{5-3} \\
& =2.500
\end{aligned}
$$

We have the second family as;

$$
\text { Then, } \quad \begin{aligned}
\quad \mathrm{b} & =16, \mathrm{~d}=2 \text { : } \\
\lambda=\frac{d}{b+d} & \cdot(1000) \\
= & \frac{16}{16+2} \cdot(1000) \\
= & \frac{16}{18} \cdot(1000) \\
= & \frac{16000}{18}
\end{aligned}
$$

$$
\mu=\frac{2}{16+2} \cdot(1000)
$$

$$
\begin{aligned}
& =\frac{2}{18} \cdot(1000) \\
& =\frac{2000}{18} \\
& =111
\end{aligned}
$$

Again the probability of extinction is given by

$$
\begin{aligned}
P_{E} & =\left(\frac{\mu}{\lambda}\right)^{n_{o}} \\
& =\left(\frac{111}{889}\right)^{1}
\end{aligned}
$$$$
=0.125
$$

From the results, number of individuals that died without offspring $=0$.

Number of individuals alive without offspring $=4$

To evaluate the mean number, we first know the number of individuals who died without offspring $\left(N_{D}\right)$ and those who are alive without offspring $\left(N_{A}\right)$. let,

$N_{d}=0, N_{a}=4$.

$$
\begin{aligned}
\text { Mean number of offspring }(\bar{x})=\frac{\text { Total number of birt } h s}{\text { Number }} & \text { of fertile parents } \\
& =\frac{T_{B}}{T_{B}-\left(N_{D}+N_{A}\right)} \\
& =\frac{16}{16-4} \\
& =\frac{16}{12} \\
& =1.333
\end{aligned}
$$

The mean number of offspring $(\bar{x})=1.33$ 


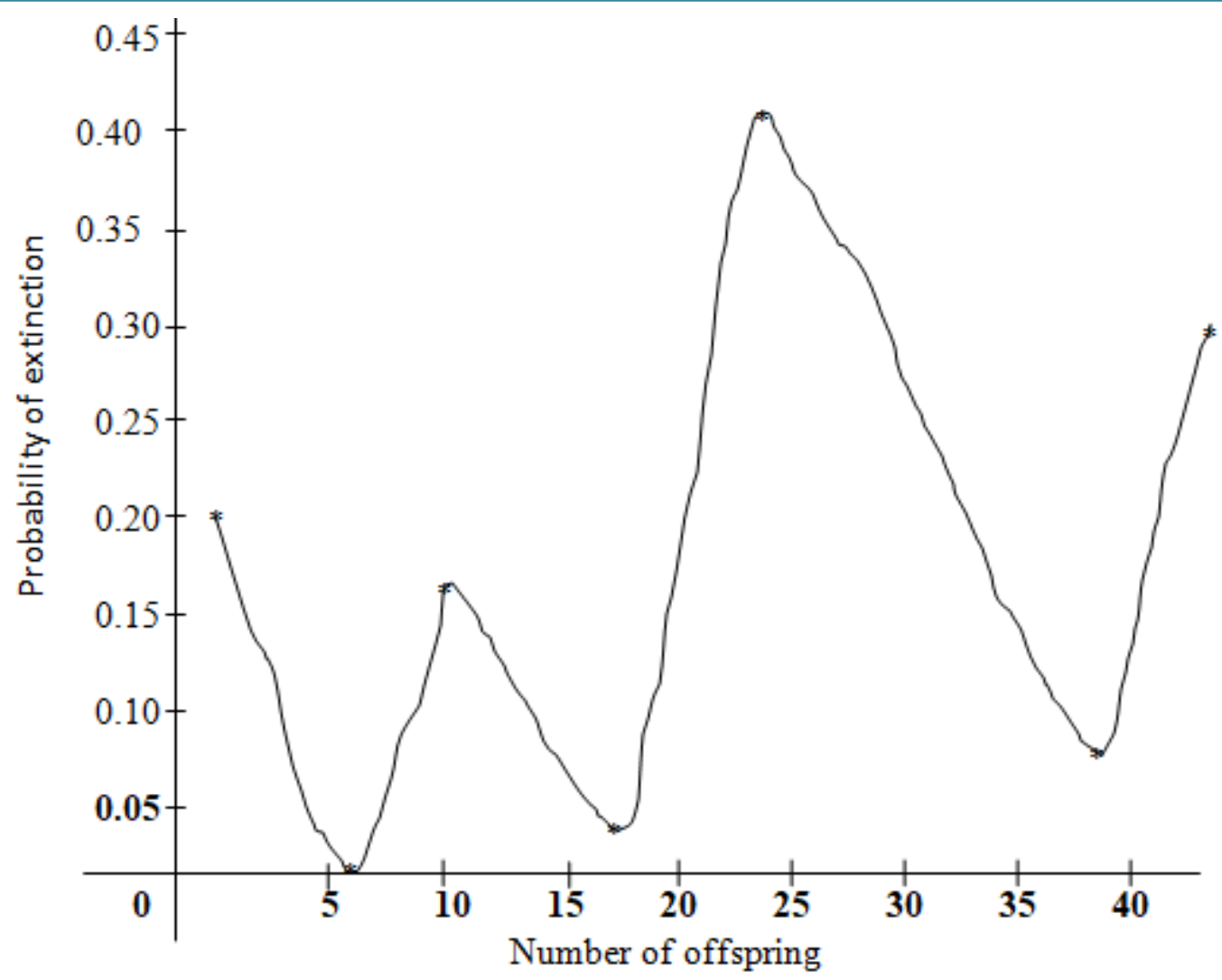

Figure 1: Graph of Extinction Probability against Number of offspring

\section{Conclusion}

We have developed a method on the stochastic differential equation for birth-death process to generate and solving extinction probability. The family with $p_{k_{17}}=0.013$ has low extinction probability rate as shown in fig $1 . p_{k_{23}}$ has the highest extinction probability and killing rate. If the killing continue at this rate, death will overtake birth while $p_{k_{6}=0=p_{24}}$ has no killing effect since death is impossible.

\section{References}

[1] Dzaan, S., Onah, E.S and Kimbir, A. R., 2009. On the extinction probability of a family name. Science world Journal. Www. Scienceworldjournal.org. 4: 1597-6343.

[2] Omotosho, M., 2014. College and University Statistics. Ibadan : Yosod Book Publisher Limited.

[3] Scott. E.C., 2006. Extinction (Biology). Microsoft@ Student 2007 [DVD].Redmond, WA : Microsoft Corporation ,2006.

[4] Fugo, T., 2013. Birth- Death models. J. Appl. Prob 6, 205-218

[5] Van Doorn, E., 2004. Representation for the rate of convergence of Birth - Death process. Theory of probability. Math statistic. 65, 37-43. 